\title{
Tricritical behavior in deterministic aperiodic Ising systems
}

\author{
T. A. S. Haddad*, Angsula Ghosh and S. R. Salinas \\ Instituto de Física, Universidade de São Paulo \\ Caixa Postal 66318, 05315-970, São Paulo, SP, Brazil
}

(February 7, 2020)

\begin{abstract}
We use a mixed-spin model, with aperiodic ferromagnetic exchange interactions and crystalline fields, to investigate the effects of deterministic geometric fluctuations on first-order transitions and tricritical phenomena. The interactions and the crystal field parameters are distributed according to some two-letter substitution rules. From a Migdal-Kadanoff real-space renormalization-group calculation, which turns out to be exact on a suitable hierarchical lattice, we show that the effects of aperiodicity are qualitatively similar for tricritical and simple critical behaviour. In particular, the fixed point associated with tricritical behaviour becomes fully unstable beyond a certain threshold dimension (which depends on the aperiodicity), and is replaced by a two-cycle that controls a weakened and temperature-depressed tricritical singularity.
\end{abstract}

The introduction of quenched disorder weakens (and sometimes eliminates) first-order transitions and tricritical singularities in the phase diagram of statistical models. In two dimensions, rigorous arguments show that any amount of disorder completely eliminates first-order transitions in ferromagnetic model systems [1]. In three dimensions, approximate real-space and perturbative renormalization-group analyses [2], as well as numerical simulations, indicate that a finite strength of disorder is required to weaken first-order transitions and depress the tricritical temperature. In particular, disordered versions of the two-dimensional ferromagnetic $q$-state Potts model [3, 4] (for $q>4$, on the square lattice, the uniform model displays a first-order transition) and the Blume-Emery-Griffiths (BEG) model [5-7] (whose uniform version displays tricritical and critical-end points), which have been thoroughly investigated, are well adjusted to this scenario. It remains unclear the important question of what are (if any) the universality classes of the disorder-induced continuous transitions in these systems (see, for example, a recent review by Cardy [8]).

Instead of looking at the (presumably) more difficult problem posed by fluctuations associated with quenched disorder, in the present publication we consider the effects on first-order transitions of the geometric fluctuations introduced by deterministic but aperiodically distributed exchange interactions. For quenched disordered interactions, the Harris criterion [9] indicates a change in the critical behaviour of simple ferromagnets whenever the critical exponent associated with the singularity of the specific heat of the underlying uniform model is positive. According to a heuristic argument of Luck [10], which has been checked in a number of cases [11, and references therein], the introduction of aperiodic interactions leads to an analogous criterion of relevance of the geometric fluctuations on the critical (second-order) behaviour. Some of us have recently shown that a similar criterion may be exactly established for ferromagnetic Potts models on Migdal-Kadanoff hierarchical lattices, with a layered distribution of exchange interactions according to a class of two-letter substitution rules [12]13. For relevant geometric fluctuations, there appears a two-cycle of the recursion relations in parameter space that gives rise to a new universality class of (aperiodic) critical behaviour [14]. Along the lines of these investigations, we introduce aperiodic interactions in a simple mixed-spin model to analyze the effects on tricritical behaviour and first-order phase boundaries. It should be mentioned that recent extensive Monte Carlo calculations indicate that the phase transition of the 8-state square-lattice Potts model is indeed driven to second order by a layered aperiodic distribution of exchange couplings [15].

Besides the better known BEG model, another simple generalization of the Ising model displaying first-order transitions and tricritical points is a mixed-spin system, given by the Hamiltonian

$$
\mathcal{H}=-\sum_{(i, j)} J_{i j} \sigma_{i} S_{j}+\sum_{j} D_{j} S_{j}^{2}
$$

where $\sigma_{i}= \pm 1$, for $i$ belonging to one sublattice of a bipartite lattice, $S_{j}= \pm 1$ or 0 , for $j$ belonging to the other sublattice, and the first sum is over nearest-neighbor sites on different sublattices. The description of this mixed-spin model demands a larger unit cell than the BEG model in zero field. This extra difficulty, however, poses no problem to the study of the model under a Migdal-Kadanoff real-space renormalization-group approximation. Moreover, we need just two scaling fields, instead of three as in the BEG model, to describe the even space of parameters. We then take advantage of this model, and of the simplicity of the Migdal-Kadanoff approximation (which turns out to be exact on a suitable hierarchical lattice), to introduce aperiodic interactions for analyzing the effects of geometric fluctuations on the main features of the phase diagram. 
To obtain the Migdal-Kadanoff recursion relations, it is convenient to rewrite Hamiltonian (1) in the equivalent form

$$
\mathcal{H}=-\sum_{(i, j)} J_{i j} \sigma_{i} S_{j}+\frac{1}{2} \sum_{(i, j)} D_{i j} \sigma_{i}^{2} S_{j}^{2} .
$$

Many (mainly approximate) results are known for the uniform $\left(J_{i j}=J, D_{i j}=D\right)$ version of this model. On a honeycomb lattice, a star-triangle transformation (summing over $S_{j}$ ) can be used to reduce the problem to an exactly soluble spin-1/2 Ising model on a simple triangular lattice, in which case, however, the temperature $\left(k_{B} T\right)$ versus "anisotropy" $(D / J)$ phase diagram presents only a line of continuous transitions [16]. For the so-called union jack lattice, an exact solution can also be found for a restricted range of parameters, by mapping the model onto an eight-vertex problem [17]. On a lattice of sufficiently high coordination, some effective-field [18] and self-consistent [19] approximations, as well as a Bethe lattice calculation [20], suggest the existence of a first-order boundary that becomes a $\lambda$ line beyond a tricritical point. A detailed Migdal-Kadanoff renormalization-group calculation 21] predicts the existence of a tricritical point on hypercubic lattices of dimension $d \gtrsim 2.1$, which precludes the case of planar lattices. This is also confirmed by renormalization-group calculations in momentum space [21], at one-loop approximation, that do support the existence of the tricritical point predicted by the Curie-Weiss version of the model. Monte Carlo [22] and numerical transfer matrix calculations [23] point out in this direction as well, with no indication of tricritical phenomena in this mixed-spin model on a square lattice.

We now consider Hamiltonian (2) and suppose that $J_{i j}$ (and $D_{i j}$ ) may assume one out of two values, $J_{A}$ or $J_{B}\left(D_{A}\right.$ or $D_{B}$ ), according to the sequence of letters generated by the iteration of a substitution rule. In this paper, we work with two distinct binary rules,

$$
\text { (i) } A \rightarrow A B B, \quad B \rightarrow A A A,
$$

and

$$
\text { (ii) } A \rightarrow A A B, \quad B \rightarrow A A A .
$$

For example, the iteration of the first rule leads to the following stages,

$$
A \rightarrow A B B \rightarrow A B B A A A A A A \rightarrow \ldots
$$

Each rule is characterized by a substitution matrix, which relates the number of letters $A$ and $B$ in one stage of the iteration with the corresponding numbers in the previous stage. For the first rule, the substitution matrix is given by

$$
\mathbf{M}=\left(\begin{array}{ll}
1 & 3 \\
2 & 0
\end{array}\right)
$$

with eigenvalues $\lambda_{1}=3$ and $\lambda_{2}=-2$. For the second rule, we have

$$
\mathbf{M}=\left(\begin{array}{ll}
2 & 3 \\
1 & 0
\end{array}\right)
$$

with eigenvalues $\lambda_{1}=3$ and $\lambda_{2}=-1$. In a given stage of the iteration, the fluctuation in the number of letters $A$ or $B$ relative to the mean number behaves asymptotically as $N^{\omega}$, where $N$ is the total number of letters in the sequence, and $\omega=\ln \left|\lambda_{2}\right| / \ln \lambda_{1}$ is a wandering exponent. We thus see that the first rule, with an wandering exponent $\omega=\ln 2 / \ln 3$, gives rise to stronger geometric fluctuations than the second one, with $\omega=0$, and should be more effective in perturbing the critical behaviour.

The Migdal-Kadanoff (MK) approximation on a $d$-dimensional hypercubic lattice turns out to be exact on the hierarchical cell shown in figure 1. For this type of cell, the MK scheme corresponds to a decimation of the two spins located along each bond, a spin- $1 / 2$ and a spin-1, followed by the moving and collapsing of $m$ such bonds. There is also a relationship, $d=1+\ln m / \ln 3$, between the number of branches $m$ and the Euclidean dimension $d$. Note that the renormalization procedure amounts to a reverse application of the substitution rule generated by the aperiodic sequence. Also, note that we can as well perform the more usual trick of bond-moving before decimation (which is also exact on a suitable hierarchical lattice), but the qualitative results should not depend on this choice.

For the first aperiodic rule, $A \rightarrow A B B, B \rightarrow A A A$, the MK procedure yields the recursion relations

$$
\begin{aligned}
K_{A}^{\prime}= & -\frac{m}{2} \ln \left\{\exp \left(K_{A}\right)+\exp \left(-K_{A}\right) \cosh \left(2 K_{B}\right)+\cosh \left(K_{B}\right) \exp \left(\frac{\Delta_{A}}{2}+\frac{\Delta_{B}}{2}\right)\right\} \\
& +\frac{m}{2} \ln \left\{\exp \left(K_{A}\right) \cosh \left(2 K_{B}\right)+\exp \left(-K_{A}\right)+\cosh \left(K_{B}\right) \exp \left(\frac{\Delta_{A}}{2}+\frac{\Delta_{B}}{2}\right)\right\},
\end{aligned}
$$




$$
\begin{aligned}
K_{B}^{\prime}= & -\frac{m}{2} \ln \left\{\exp \left(K_{A}\right)+\exp \left(-K_{A}\right) \cosh \left(2 K_{A}\right)+\cosh \left(K_{A}\right) \exp \left(\Delta_{A}\right)\right\} \\
+ & \frac{m}{2} \ln \left\{\exp \left(K_{A}\right) \cosh \left(2 K_{A}\right)+\exp \left(-K_{A}\right)+\cosh \left(K_{A}\right) \exp \left(\Delta_{A}\right)\right\} \\
\Delta_{A}^{\prime}= & -m \ln \left\{\exp \left(-\Delta_{B}\right)\right. \\
\times & {\left[\exp \left(K_{A}\right)+\exp \left(-K_{A}\right) \cosh \left(2 K_{B}\right)+\cosh \left(K_{B}\right) \exp \left(\frac{\Delta_{A}}{2}+\frac{\Delta_{B}}{2}\right)\right] } \\
\times & {\left[\exp \left(K_{A}\right) \cosh \left(2 K_{B}\right)+\exp \left(-K_{A}\right)+\cosh \left(K_{B}\right) \exp \left(\frac{\Delta_{A}}{2}+\frac{\Delta_{B}}{2}\right)\right] } \\
\times & {\left.\left[2 \cosh \left(K_{A}\right) \cosh \left(K_{B}\right)+\exp \left(\frac{\Delta_{A}}{2}+\frac{\Delta_{B}}{2}\right)\right]^{-2}\right\} } \\
\Delta_{B}^{\prime}= & -m \ln \left\{\exp \left(-\Delta_{A}\right)\right. \\
& \times\left[\exp \left(K_{A}\right)+\exp \left(-K_{A}\right) \cosh \left(2 K_{A}\right)+\cosh \left(K_{A}\right) \exp \left(\Delta_{A}\right)\right] \\
& \times\left[\exp \left(K_{A}\right) \cosh \left(2 K_{A}\right)+\exp \left(-K_{A}\right)+\cosh \left(K_{A}\right) \exp \left(\Delta_{A}\right)\right] \\
& \left.\times\left[2 \cosh \left(K_{A}\right)+\exp \left(\Delta_{A}\right)\right]^{-2}\right\}
\end{aligned}
$$

where $K_{A, B}=\beta J_{A, B}$ and $\Delta_{A, B}=\beta D_{A, B}$, with $\beta=1 / k_{B} T$. For all values of $m \geq 1$, these recursion relations have a set of trivial fixed points, given by

$$
P_{+} \equiv\left(K_{A}^{*}, K_{B}^{*}, \Delta_{A}^{*}, \Delta_{B}^{*}\right)=(0,0,-\infty,-\infty),
$$

which is a sink of high-density paramagnetic phase (where the density is related to the mean value $\left\langle S_{i}^{2}\right\rangle$, so that low density means the predominance of spin 0),

$$
P_{-} \equiv\left(K_{A}^{*}, K_{B}^{*}, \Delta_{A}^{*}, \Delta_{B}^{*}\right)=(0,0, \infty, \infty),
$$

which is a sink of low-density paramagnetic phase,

$$
O \equiv\left(K_{A}^{*}, K_{B}^{*}, \Delta_{A}^{*}, \Delta_{B}^{*}\right)=(0,0,0,0),
$$

corresponding to the high-temperature boundary between the paramagnetic phases,

$$
F_{+} \equiv\left(K_{A}^{*}, K_{B}^{*}, \Delta_{A}^{*}, \Delta_{B}^{*}\right)=(\infty, \infty,-\infty,-\infty),
$$

associated with a zero-temperature high-density ferromagnetic phase, and

$$
F_{-} \equiv\left(K_{A}^{*}, K_{B}^{*}, \Delta_{A}^{*}, \Delta_{B}^{*}\right)=(\infty, \infty, \infty, \infty)
$$

with $\Delta_{A, B}^{*} / K_{A, B}^{*}=2$, which corresponds to a zero-temperature low-density ferromagnetic phase.

For $m>1$ (which corresponds to $d>1$ ), there is a non-trivial fixed point at $\Delta_{A}^{*}=\Delta_{B}^{*}=-\infty$, for $K_{A}^{*}=K_{B}^{*}$ finite. This fixed point, which we shall call $I$, is associated with the critical behaviour of the simple spin-1/2 Ising model, since fixing the crystal field (biquadratic exchange) at $-\infty$ completely prevents the $S$ spins to assume 0 values. In this spin-1/2 space, it should be pointed out that the recursion relations also present a two-cycle (that is, a set of two fixed points of the second-iterate). As discussed in a recent publication [14], this two-cycle is associated with the new universality class of the aperiodic ferromagnetic Ising model. Indeed, the critical behaviour of the Ising model on the hierarchical lattice underlying the MK approximation, and with aperiodic interactions according to the rule $A \rightarrow A B B, B \rightarrow A A A$, is controlled by this two-cycle, the singularity being weaker as compared with the uniform (periodic) model.

For $m \gtrsim 3.33$ (numerical evidence points in fact to $10 / 3$ ), corresponding to $d \gtrsim 2.1$ (or $\ln 10 / \ln 3=2.0959 \ldots$, as suggested by the numerical calculations), there appear two novel non-trivial fixed points. In the uniform case $\left(J_{A}=J_{B}, D_{A}=D_{B}\right)$, Quadros and Salinas [21] have shown that one of them is a discontinuity fixed point, associated with a first-order phase transition (according to an application of the Nienhuis and Nauenberg criterion for the identification of discontinuity fixed points). On the basis of a detailed analysis of the connectivity of the flow lines in parameter space, the other fixed point of the uniform model was shown [21] to be associated with the tricritical 
behaviour. In the uniform case, the discontinuity fixed point displays a saddle character, with an attractive manifold emerging from the zero-temperature high-density ferromagnetic trivial fixed point, and from the tricritical fixed point (see figure 2, where a schematic view of the flows is presented). The repulsive directions flow towards the sink of the low-density paramagnetic phase, and towards the zero-temperature low-density ferromagnetic trivial fixed point. The stable manifold is thus associated with a first order line. The fully unstable tricritical fixed point is connected with the Ising, $D=-\infty$, fixed point, giving rise to a second-order boundary.

In the present aperiodic case, for $m \gtrsim 3.33$, these two fixed points still appear in the $K_{A}=K_{B}, \Delta_{A}=\Delta_{B}$, subspace, but since the parameter space is four-dimensional we have to be careful to generalize the overall picture of the last paragraph. In fact, we should pay attention to the fact that now there are four scaling fields: the reduced temperature, two crystal fields (instead of only one, as in the uniform case), and the strength of the aperiodicity, as measured, for example, by the ratio $r=J_{A} / J_{B}$. This last scaling field, which is of completely different nature, is the determinant factor for the analysis of stability of the fixed points against aperiodicity. If it turns out to be relevant around a certain fixed point, it means that this fixed point cannot be reached unless $r$ assumes a well-defined value, usually unity, so that any amount of aperiodicity changes the critical behaviour controlled by this particular fixed point. On the other hand, irrelevancy of this scaling field suggests that, whatever the strength of the geometric fluctuations, this fixed point continues to control the critical behaviour.

Consider now $m=3.4$ (dimension $d \approx 2.1$ ). The fixed points are at $K_{A}^{*}=K_{B}^{*}=1.5248 \ldots, \Delta_{A}^{*}=\Delta_{B}^{*}=2.2118 \ldots$ (discontinuity fixed point in the uniform model) and $K_{A}^{*}=K_{B}^{*}=1.1447 \ldots, \Delta_{A}^{*}=\Delta_{B}^{*}=1.3953 \ldots$ (tricritical fixed point of the uniform case). The linearization of the recursion relations (6)-(96), in the neighborhood of this fixed point lead to the eigenvalues

$$
\Lambda_{1}=4.3687 \ldots, \Lambda_{2}=0.7705 \ldots, \Lambda_{3}=-3.8520 \ldots, \Lambda_{4}=-0.5825 \ldots
$$

The first two eigenvalues are the same as in the uniform case. The modulus of the last two eigenvalues indicates the preservation of the saddle character of this fixed point. The linearization about the second fixed point yields the eigenvalues

$$
\Lambda_{1}=4.1671 \ldots, \Lambda_{2}=1.2360 \ldots, \Lambda_{3}=-3.7346 \ldots, \Lambda_{4}=-0.9194 \ldots
$$

Again, $\Lambda_{1}$ and $\Lambda_{2}$ assume the same values of the uniform case. The modulus of the third eigenvalue is larger than 1 , so far preserving the unstable character of the uniform tricritical fixed point. The last eigenvalue, however, indicates an attractive direction towards this fixed point, in contrast to the uniform model, in which case the tricritical fixed point is fully unstable. However, we have already remarked that a given characteristic fixed point associated with the uniform model still controls the critical behaviour of the aperiodic system only if the strength of the aperiodicity is an irrelevant scaling field. This is precisely what is happening in this case. The modulus of $\Lambda_{4}$ asserts that the tricritical fixed point associated with the uniform model can be reached, even in the presence of aperiodicity.

An analysis of the flow lines of the recursion relations in parameter space fully supports the idea that these two fixed points continue to perform exactly the same functions in the aperiodic as well as in the uniform case. In other words, the first one is indeed a discontinuity fixed point, and the other one is associated with the tricritical behaviour. In a phase diagram consisting of temperature, crystal fields and the aperiodicity ratio $r$, there exists then a line of tricritical points extending along the $r$ direction. Finally, we note that $\Lambda_{3}$ and $\Lambda_{4}$ are negative, for both fixed points. This is a common situation for these aperiodic systems [14], which reflects a flipping approximation to (or furthering from) the fixed points, and is related to the discrete nature of the renormalization procedure and to the existence of two competing energy scales, $A$ and $B$.

For larger dimensions (larger values of $m$ ), this whole picture is changed. Take, for example, $m=9$, corresponding to three dimensions. The discontinuity fixed point of the uniform model is located at $K_{A}^{*}=K_{B}^{*}=4.8427 \ldots, \Delta_{A}^{*}=$ $\Delta_{B}^{*}=9.0255 \ldots$. The tricritical fixed point is located at $K_{A}^{*}=K_{B}^{*}=0.4514 \ldots, \Delta_{A}^{*}=\Delta_{B}^{*}=0.2190 \ldots$. The linearization of the recursion relations in the neighborhood of these fixed points lead to the eigenvalues

$$
\Lambda_{1}=12.0154 \ldots, \Lambda_{2}=0.0032 \ldots, \Lambda_{3}=-10.5075 \ldots, \Lambda_{4}=-0.0002 \ldots
$$

around the discontinuity fixed point (the first two eingenvalues are the same as in the uniform case), and

$$
\Lambda_{1}=9.1600 \ldots, \Lambda_{2}=2.5865 \ldots, \Lambda_{3}=-9.0707 \ldots, \Lambda_{4}=-1.7413 \ldots
$$

around the second fixed point (again, the first two eingenvalues are the same as in the uniform case). Note that this time the modulus of all of the eigenvalues of the uniform tricritical fixed point are larger than unity, which means that it is fully unstable against any aperiodic perturbations. Hence, it cannot be reached whatever the strength of the geometric fluctuations, except in the trivial, uniform case, $J_{A}=J_{B}, D_{A}=D_{B}$. As in the case of the spin- $1 / 2$ 
critical fixed point $\left(D_{A}=D_{B}=-\infty\right)$, there also appears a two-cycle of the recursion relations. This two-cycle is located at

$$
\left(K_{A}^{*}, K_{B}^{*}, \Delta_{A}^{*}, \Delta_{B}^{*}\right)_{1}=(0.3759 \ldots, 4.5304 \ldots, 0.1472 \ldots, 8.0027 \ldots)
$$

and

$$
\left(K_{A}^{*}, K_{B}^{*}, \Delta_{A}^{*}, \Delta_{B}^{*}\right)_{2}=(2.0653 \ldots, 0.2772 \ldots, 3.4221 \ldots, 0.0905 \ldots) .
$$

As discussed in a previous publication [14], we should now study the behaviour of the second iterate of the recursion relations around any one of the points belonging to the two-cycle. From the linearization of the second iterates of the recursion relations about these points, we have the eigenvalues

$$
\Lambda_{1}=114.3038 \ldots, \Lambda_{2}=82.0353 \ldots, \Lambda_{3}=5.1867 \ldots, \Lambda_{4}=0.0014 \ldots
$$

Now there is at least one eigenvalue with modulus less than 1, which guarantees that the two-cycle is physically accessible. From a numerical analysis of the connectivity of the flow lines in parameter space, we can check that this two-cycle is indeed associated with the tricritical behaviour (in analogy with the second-order transitions associated with the two-cycle in the spin-1/2 subspace).

For $m=27$, corresponding to $d=4$, we have the same general features. The uniform tricritical fixed point is fully unstable, and there appears a two-cycle, which is presumably associated with a novel tricritical behaviour. Numerical calculations point out that the two-cycle appears as a kind of bifurcation of the uniform tricritical fixed point, at $m \approx 3.45$ (corresponding to $d \approx 2.13$ ). Further numerical work shows that, for a given ratio $J_{A} / J_{B}$, the temperature that locates the system inside the basin of attraction of the two-cycle (that is, the tricritical temperature) is systematically lower as compared with the tricritical temperature of the uniform model (and thus leads to a smaller first-order region in the phase diagram). These features are also generally present in disordered tricritical systems in three dimensions [5].

For the aperiodic rule $A \rightarrow A A B, B \rightarrow A A A$, with a smaller wandering exponent $(\omega=0$ in comparison with $\omega=\ln 2 / \ln 3$ for the previous sequence), we have a different set of recursion relations, but the results are similar. In this case, the calculations show that the uniform tricritical fixed point remains accessible (there is one eigenvalue with modulus less than 1) whatever the value of $m$. The smaller eigenvalue around the tricritical fixed point approaches unity as $m$ goes to infinity. Weaker geometric fluctuations are therefore unable to relevantly perturb the system, whose multicritical behaviour remains unchanged with respect to the uniform model.

In conclusion, we have studied the effects of deterministic aperiodicity on a simple system that displays first and second-order transition lines and a tricritical point. Using a simple Migdal-Kadanoff approximation, we show that a certain class of non-random geometric fluctuations may change the tricritical behaviour (by turning into a fully unstable node the fixed point associated with the uniform tricritical behaviour). There is then a two-cycle of the recursion relations that is shown to control the tricritical behaviour. Numerical calculations indicate a depression of the tricritical temperatures (as it used to happen in the presence of quenched disorder). In spite of the limitations of the MK approximation (or, alternatively, the artificiality of the hierarchical lattices in which it turns out to be exact), the results of this investigation provide suggestions for the analysis of multicritical behaviour in similar systems on realistic Bravais lattices.

The authors wish to thank A. P. Vieira for useful discussions. This work has been supported by the Brazilian agency FAPESP.

* e-mail address: thaddad@if.usp.br

[1] M. Aizenman and J. Wehr, Phys. Rev. Lett. 62, 2503 (1989).

[2] K. Hui and A. N. Berker, Phys. Rev. Lett. 62, 2507 (1989).

[3] S. Chen, A. M. Ferrenberg and D. P. Landau, Phys. Rev. Lett. 69, 1213 (1992).

[4] J. L. Cardy and J. L. Jacobsen, Phys. Rev. Lett.79, 4063 (1997).

[5] A. Falicov and A. N. Berker, Phys. Rev. Lett. 76, 4380 (1996).

[6] A. Kabakçioglu and A. N. Berker, Phys. Rev. Lett. 82, 2572 (1999).

[7] N. S. Branco and B. Boechat, Phys. Rev. B 56, 11673 (1997).

[8] J. L. Cardy, Physica A 263, 215 (1999). 
[9] A. B. Harris, J. Phys. C 7, 1671 (1974).

[10] J. M. Luck, Europhys. Lett. 24, 359 (1993).

[11] U. Grimm and M. Baake, "Aperiodic Ising Models". In: R. V. Moody (ed.), The Mathematics of Long Range Aperiodic Order, Kluwer, Dordrecht, p. 199 (1997).

[12] S. T. R. Pinho, T. A. S. Haddad and S. R. Salinas, Physica A 257, 515 (1998).

[13] A. C. N. de Magalhães, S. R. Salinas and C. Tsallis, J. Phys. A: Math. Gen. 31, L567 (1998).

[14] T. A. S. Haddad, S. T. R. Pinho and S. R. Salinas, Phys. Rev. E 61, 3330 (2000).

[15] P. E. Berche, Ch. Chatelain and B. Berche, Phys. Rev. Lett. 80, 297 (1998).

[16] L. L. Gonçalves, Phys. Scr. 32, 248 (1985).

[17] A. Lipowski and T. Horiguchi, J. Phys. A: Math. Gen. 28, L261 (1995).

[18] T. Kaneyoshi, J. Phys. Soc. Jpn. 56, 2675 (1987).

[19] N. Benayad, A. Klümper, J. Zittartz and A. Benyoussef, Zeits. Phys. B 77, 339 (1989).

[20] N. R. da Silva and S. R. Salinas, Phys. Rev. B 44852 (1991).

[21] S. G. A. Quadros and S. R. Salinas, Physica A 206, 479 (1994).

[22] G. M. Zhang and C. Z. Yang, Phys. Rev. B 48, 9452 (1993).

[23] G. M. Buendía and M. A. Novotny, J. Phys.: Condens. Matter 9, 5951 (1997). 


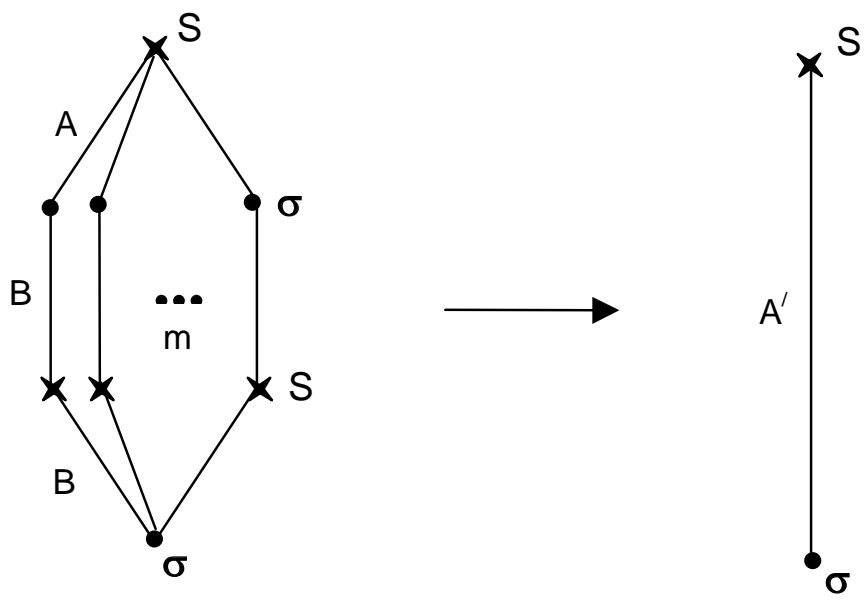

FIG. 1. Example of the hierarchical cell representing part of the Migdal-Kadanoff decimation scheme. Crosses correspond to spins-1, and dots to spins-1/2. By summing over the two internal spins of each of the $m$ branches, we obtain $K_{A}^{\prime}$ or $\Delta_{A}^{\prime}$.

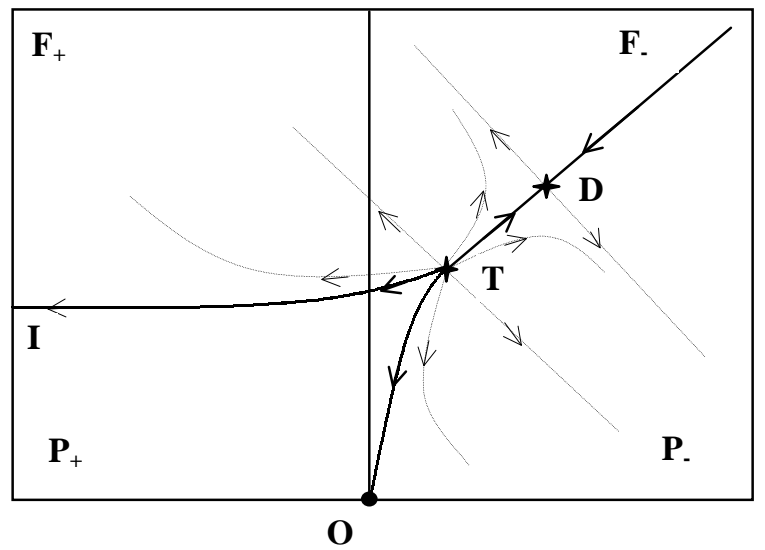

FIG. 2. Schematic view of the renormalization-group flows in parameter space, for the uniform version of the mixed-spin Ising model, with $K$ in the vertical axis, and $\Delta$ in the horizontal. $T$ is the tricritical fixed point, and $D$ is the discontinuity one. 